prophylactic agent of non-epileptic events, partially effective in $74 \%$. AEDs had no significant effect on non-paroxysmal events and only occasional control of paroxysmal symptoms. Diazepam sometimes reduced the duration of dystonic attacks. (Panagiotakaki E, Gobbi G, Neville B, et al. Evidence of a non-progressive course of alternating hemiplegia of childhood: study of a large cohort of children and adults. Brain December 2010;133:3598-3610). (Respond: Pr Alexis Arzimanoglou, Institute for Children and Adolescents with Epilepsy, Hopital Femme Mere Enfant, Hospices Civils de Lyon, 59 Boulevard Pinel, 69677, Lyon, France. E-mail: aarzimanoglou@orange.fr).

COMMENT. AHC is characterized by episodes of hemiplegia, dystonic and other non-epileptic paroxysmal events (paroxysmal nystagmus, autonomic disorder), epileptic seizures, and global neurological impairment. Between episodes, patients are neurologically impaired, with ataxia, athetosis or chorea, the majority developing mental retardation. Onset is in infancy, sometimes in the neonatal period. Consciousness is preserved during episodes. The pathophysiology has been compared to hemiplegic migraine, but differs in the development of fixed neurological impairment and retardation.

\title{
NEONATAL DISORDERS
}

\section{HYPOXIC-ISCHEMIC-ENCEPHALOPATHY STUDIED BY EEG AND MR SPECTROSCOPY}

Amplitude-integrated EEG (a-EEG) time course during the first 24 hrs of life was related to brain metabolism changes detected by proton MR spectroscopy (H-MRS) at 710 days of post-natal life in non-cooled term newborns with hypoxic-ischemicencephalopathy (HIE). In a study at the Neonatology Unit, University of Bologna, Italy, a-EEG at 6, 12 and 24 hrs of life in 27 of 31 patients who survived was correlated with outcome; the a-EEG showed improvement in newborns with normal H-MRS and good outcome and a deterioration in those with abnormal H-MRS and poor outcome. a-EEG time course in the first 24 hrs of life may document the severity and evolution of cerebral damage following a perinatal IH event. Both H-MRS and a-EEG show a good correlation with outcome. (Ancora G, Soffritti S, Lodi R, et al. A combined a-EEG and MR spectroscopy study in term newborns with hypoxic-ischemic-encephalopathy. Brain Dev 2010;32:835-842). (Respond: E-mail: gina.ancora@unibo.it).

COMMENT. Prompt evaluation of newborns with HI encephalopathy to determine severity of brain damage and prognosis is important in therapy and follow-up. Seizure activity detected by a-EEG was associated with poor outcome only in patients with abnormal a-EEG background pattern. A normal a-EEG background at $24 \mathrm{hrs}$ of life was predictive of normal outcome, a finding in agreement with that of van Rooij et al. 2005 (cited by authors), who reported that the background activity at onset of seizures is the best predictor of outcome. H-MRS shows a better correlation with outcome compared to conventional MRI. a-EEG and H-MRS have a similar sensitivity and specificity for prediction of outcome. 